\title{
XMM-Newton observations of the eastern jet of SS 433
}

\author{
W. Brinkmann ${ }^{1}$, G. W. Pratt ${ }^{1}$, S. Rohr ${ }^{1}$, N. Kawai ${ }^{2}$, and V. Burwitz ${ }^{1}$ \\ 1 Max-Planck-Institut für Extraterrestrische Physik, Giessenbachstrasse, 85740 Garching, Germany \\ e-mail: wpb@mpe.mpg.de \\ 2 Tokyo Institute of Technology, 2-12-1 O-okayama, Meguro-ku, 152-8551 Tokyo, Japan
}

Received 8 May 2006 / Accepted 26 October 2006

\section{ABSTRACT}

\begin{abstract}
Context. The radio supernova remnant W50 hosts at its center the peculiar galactic X-ray binary SS 433. It shows a central spherical structure with two "ears" which are supposed to be formed by the interaction of the precessing jets of SS 433 with the supernova shell. Aims. A study of the spectral and spatial structure of the X-ray emission can reveal the physical conditions of the interaction of the precessing jet of SS 433 with the material of the surrounding supernova remnant W50.

Methods. In two pointings in September/October 2004 for $30 \mathrm{ks}$ each the eastern jet of SS 433 was observed with XMM-Newton to study the outermost parts of the "ear" and the X-ray bright emission region about 35' from SS 433.

Results. The spectra consist of two components: a non-thermal power law with photon index $\Gamma \sim 2.17 \pm 0.02$ and a thermal component at a typical temperature of $k T \sim 0.3 \mathrm{keV}$. The X-ray emission seems to fill the whole interior region of the radio remnant W50. The jet terminates in the eastern "ear" in a ring-like terminal shock which indicates a flow with a kind of hollow-cone morphology. The spatial coincidence of X-ray and radio emission suggests physical conditions similar to those found at the outer shocks of ordinary supernova remnants. The bright emission region closer to SS 433 radiates non-thermally in a spatially well confined geometry at higher X-ray energies. At soft X-rays the shape of the region gets blurred, centered on the hard lenticular emission. The shape of this region and the bend in the jet propagation direction might be caused by the interaction of a re-collimated jet with the outer, non homogeneous interstellar matter distribution.

Conclusions. The physical conditions leading to the re-collimation of the jet and the peculiar emission morphology are far from being understood and require deeper observations as well as a detailed modeling of the interaction of a jet with its surroundings.
\end{abstract}

Key words. ISM: supernova remnants - ISM: jets and ourtflows - X-rays: general

\section{Introduction}

The Galactic binary SS 433 emits two oppositely directed jets with velocities of $v_{j} \sim 0.26 \mathrm{c}$, which precess under an angle of $\theta=19.8^{\circ}$ with a period of $P_{\mathrm{p}}=162.5$ days (Margon 1984). The kinetic energy of the jets is enormous and might well be in excess of $\sim 10^{40} \mathrm{erg} \mathrm{s}^{-1}$. SS 433 is thus regarded as one of the most prominent objects of the class of galactic Microquasars (Mirabel \& Rodriguez 1998).

VLA observations confirmed the distance of the object of $5 \mathrm{kpc}$ and revealed further evidence for the precessional motion of the matter of the jets (Hjellming \& Johnston 1981). Doppler shifted iron lines in the spectrum of SS433 were detected by X-ray observations with the EXOSAT satellite (Watson et al. 1986). As the centroids of the lines follow the precessional Doppler curve (see e.g. Margon 1984) the X-rays are most likely originating from the base of the jets, emitting thermal radiation at very high temperatures. In a series of Ginga observations from 1987 to 1991 (Kawai et al. 1989; Brinkmann et al. 1989; Brinkmann et al. 1991; Yuan et al. 1995) and later deep ASCA observations (Kawai et al. 1994; Kotani et al. 1996) the general picture of hydrodynamically out-flowing cooling jets was confirmed. Recent observations with the high spectral resolution of the Chandra transmission gratings (Marshall et al. 2002; Namiki et al. 2003) and the high sensitivity and broad energy band of XMM-Newton (Brinkmann et al. 2005) have put more stringent constraints on the physical parameters of the jets and on the geometrical conditions of the emission region.
The general picture is that the matter at the base of the outflowing jet is hot, $k T \sim 20 \mathrm{keV}$, and cools to low temperatures over a distance of typically a few $\times 10^{12} \mathrm{~cm}$, giving further out rise to the observed optical line emission. The emitted X-ray radiation, with a luminosity of $\sim 3 \times 10^{35} \mathrm{erg} \mathrm{s}^{-1}$, is "driven" by the thermal energy of the out-flowing gas. The much higher (by a factor of $\lesssim 10^{5}$ ) kinetic luminosity of the jets remains invisible and feeds the surrounding supernova remnant W50.

Hydrodynamic models based on this "canonical" jet model generally provide good fits to the data but might have to be refined to accommodate new recent observations. Utilizing the high spatial resolution of the Chandra ACIS instrument Doppler shifted iron lines were found at distances of $\gtrsim 10^{17} \mathrm{~cm}$ from the central source (Migliari et al. 2002). The required in-situ re-heating of the emitting gas and the temporal variability of the arcsec-scale X-ray jet during the multiple Chandra observations might be explained by a second, faster outflow in the jet (Migliari et al. 2005). Noticeable variations of the Doppler shifts of the jets' emission lines on time scales less than a day might be caused by changes in the jets' terminal velocities which are affected by environmental effects that disturb the direction or the speed of the jet (Marshall et al. 2005).

At larger distances from the compact object the matter flow of the jets interacts with the circumstellar medium and loses its energy and momentum. The radio shape of W50 shows two wellknown lobes, the so-called "ears", caused by the out-flowing jets, which seem to be confined to the shell of the host supernova remnant W50. The out-flowing matter, however, can be seen 
only very close to SS433, on scales of $\lesssim 3^{\prime \prime}$ (Blundell \& Bowler 2004) and further out, at the "ears". In between, there are no radio contours indicating the presence of a directed, collimated flow. X-ray observations with the Einstein IPC detector (Watson et al. 1983) demonstrated that the energy requirements and the morphology of the lobes are consistent with steady beaming over the life time of the remnant with the precessing jets supplying the directed energy. The emission appears patchy, peaks at distances of $\sim 35$ arcmin from the source and shows hardly any flux close to SS 433. The main contribution to the flux comes from the narrow cone of the jets of about 20 degrees opening angle, but there is, at a lower level, a much wider component suggesting that energy is "leaking out" from the well confined jets. The soft $(0.5-4.5 \mathrm{keV}) \mathrm{X}$-ray luminosity from each lobe is $\sim 6 \times 10^{34} \mathrm{ergs} \mathrm{s}^{-1}$ and their spectra are considerably softer than SS433 itself. However, the IPC data did not allow the distinction between a thermal or a non-thermal origin of the emission.

ASCA observations of the eastern jet showed that the spectrum is non thermal and best fitted by a power law with photon index $\Gamma$ in the range $\sim 1.4-2.2$ (Yamauchi et al. 1994). Further, there were indications that the spectrum hardens towards SS 433.

Two observations with the ROSAT PSPC (Brinkmann et al. 1996, Paper I) provided an X-ray image of unprecedented detail. The morphology of the eastern and the western jet appears to be quite different, which was attributed to different conditions in the circumstellar medium interacting with the jets. For the bright emission region of the eastern jet, about 35' away from SS 433, a power law form of the spectrum was confirmed. The terminal shock of the eastern jet, about $80^{\prime}$ from the central source, seemed to have a thermal spectrum with a $k T \sim 0.2 \mathrm{keV}$. However, the data quality there was rather low and the spatial structures were partly hidden by the inner support structure of the PSPC detector. We have therefore utilized the high sensitivity, high spectral resolution and wide band pass of the EPIC cameras on board XMM-Newton to perform two pointings on the eastern jet.

The outline of the paper is as follows. In the next section we give an overview over the XMM-Newton observations and describe in some detail specific aspects of the data analysis. In Sect. 3 we discuss the spectral analysis of the jets and in Sect. 4 we compare the X-ray images with the radio structure of W50. Then, in Sect. 5, we discuss the implications of the results for the physical properties of the jets and, finally, summarize the results and open questions.

\section{Observations and data analysis}

The eastern jet of W50 was observed by XMM-Newton in two pointings of $\sim 30 \mathrm{ks}$ each. On September 30, 2004 the telescopes were pointed at the position $\mathrm{RA}(\mathrm{J} 2000)$ : $19^{\mathrm{h}} 14^{\mathrm{m}} 12^{\mathrm{s}}$, Dec: $04^{\circ} 55^{\prime} 47^{\prime \prime}$, i.e. the bright maximum of X-ray emission from the eastern jet about $35^{\prime}$ east of SS 433. The outer parts of the eastern jet at RA(J2000): $19^{\mathrm{h}} 15^{\mathrm{m}} 55^{\mathrm{s}}$, Dec: $04^{\circ} 51^{\prime} 20^{\prime \prime}$ were observed on October 04, 2004. The pointings were chosen such that the entire known X-ray emitting region (Brinkmann et al. 1995) was covered. The EPIC PN camera was operated in Full Window mode with a medium filter. The MOS cameras were operated in Full Window mode, both with a medium filter as well. Although the two RGS instruments were in operation, the weak extended sources give insufficient signal for a meaningful spectral analysis.

The EPIC data were reprocessed with the XMMSAS version 6.5. The background count rates in both observations were rather low apart from one (two) small, shorter flares. The data from these periods were discarded from the spectral analysis. For the EPIC spectral analysis we selected photons with PATTERN $\leq 4$ and FLAG $=0$ for the PN and with PATTERN $\leq$ 12 and $\mathrm{FLAG}=0$ for the MOS cameras, both in the energy range from $300 \mathrm{eV}$ to $10 \mathrm{keV}$. To correct the event files for vignetting, we add a WEIGHT column to the event files using the SAS task evigweight. All subsequent science products were extracted from this column, as described in Arnaud et al. (2001).

The spectral analysis of extended structures, filling the whole field of view of the detector, represents a major challenge because of the difficulty to define a true background region. Secondly, in this observation, the pointing directions of the telescope were such that the bright structures of the X-ray emitting region fell on the central regions of the EPIC cameras. Given the well-known spatial distribution of the $\mathrm{Cu} \mathrm{K}_{\alpha}$ and Ni $\mathrm{K}_{\alpha}$ fluorescent lines in the EPN, using a background region from a different part of the detector introduces added uncertainties.

The basic background files used for the spectral analysis are the blank-sky files accumulated by Read \& Ponman (2003). These files were flare-filtered and events were selected according to the same PATTERN and FLAG criteria as the source observations, and a WEIGHT column was added to correct for vignetting. The blank-sky backgrounds were then recast onto the sky using the aspect information from the SS 433 pointings, enabling extraction of source and background spectra from the same region of the detector. The EPIC background is dominated by charged-particle events above $\sim 2 \mathrm{keV}$. The intensity of this component can vary by typically $\pm 10 \%$, and must be accounted for by renormalisation. The background renormalisation factor for each observation was calculated in the sourcefree [10-12]/[12-14] keV (EMOS/EPN) energy band, and the WEIGHT column in each background file was adjusted accordingly. Renormalisation factors for these observations are $\pm 5 \%$ for EMOS and $<20 \%$ for EPN.

Spectra were extracted from the same region in the source and background event files using the WEIGHT column. These spectra were vignetting corrected, thus effective area and response files corresponding to the on-axis position were used. These were generated using arfgen and rmfgen, respectively.

\subsection{The overall structure}

In Fig. 1 we show the total field covered by these two observations in the $0.5-10 \mathrm{keV}$ energy range. The data from the PN and the two MOS cameras were merged, exposure and vignetting corrected, and smoothed with a Gaussian filter (details of this procedure can be found in Pietsch et al. 2004). The contour lines represent the outer boundary of the radio remnant W50 obtained from the $20 \mathrm{~cm}$ VLA observations of Dubner et al. (1998). The data show that the interior of the remnant is filled with weak $\mathrm{X}$-ray emission; no X-ray flux is detected from outside the radio structure. However, much deeper X-ray observations are required to unambiguously confirm the exact spatial correlation between the outer boundaries of the radio- and X-ray emission. While, as already noted previously in Paper I, the bright X-ray emission seems not to coincide with any radio structures and is even situated in a region of low radio surface brightness, the region near the terminal shock shows features arguing for a common spatial origin of the radio and X-ray emission.

The dashed black line represents the locus from the centre of the emission structures from the terminal jet to the position of SS 433 , which lies outside of the right-hand boundary of the figure. It forms an angle of $\sim 8^{\circ}$ with the line of constant declination. In Fig. 2 we plot the (relative) intensity of the emission 


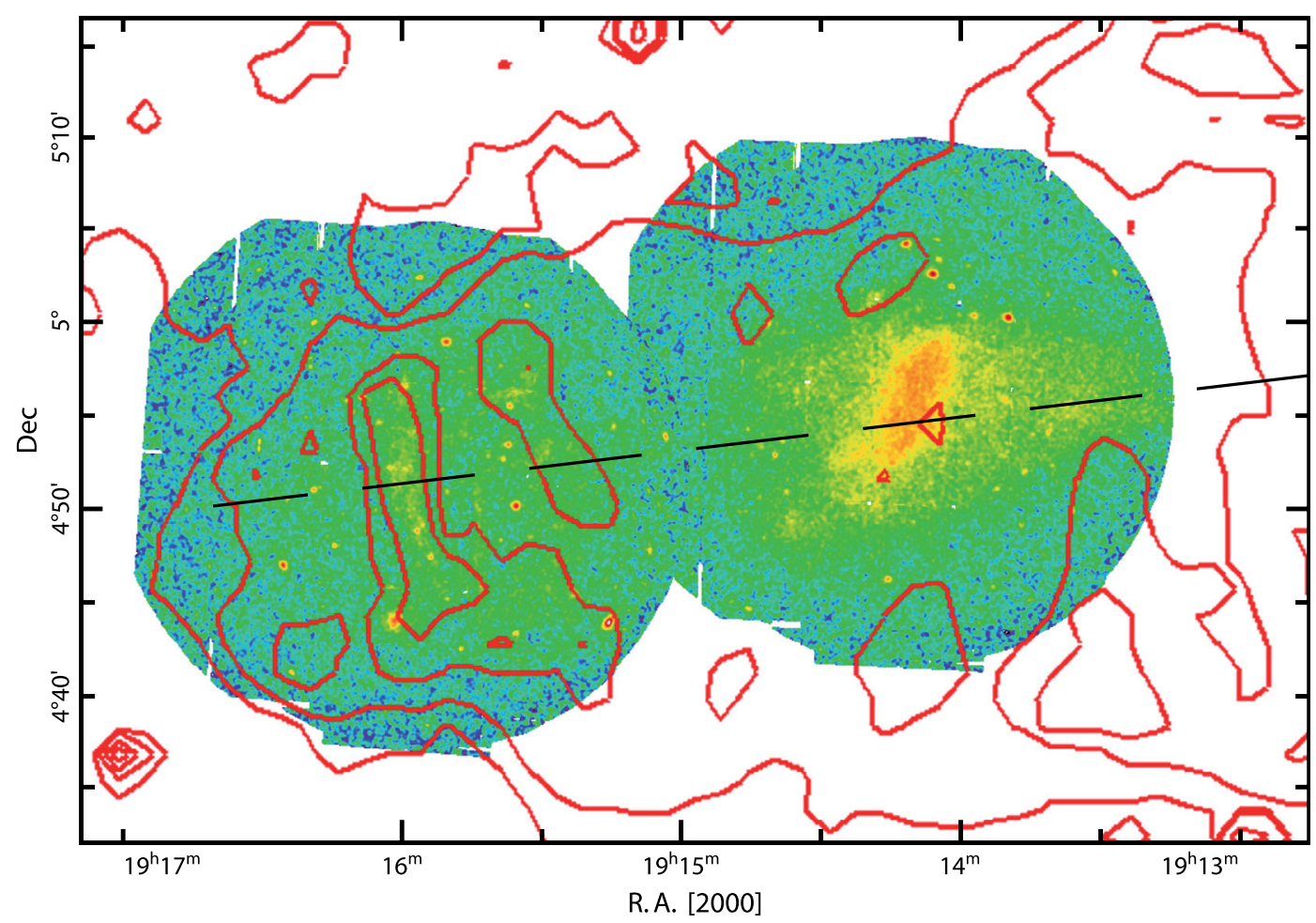

Fig. 1. Background and exposure corrected, smoothed image of the merged PN + MOS observations of the eastern jet in the $0.3-10 \mathrm{keV}$ energy band superimposed on the VLA radio contours of the outer boundaries of the eastern "ear" of W50. The dashed line represents the approximate locus from the center of the terminal shock to the position of SS 433 .

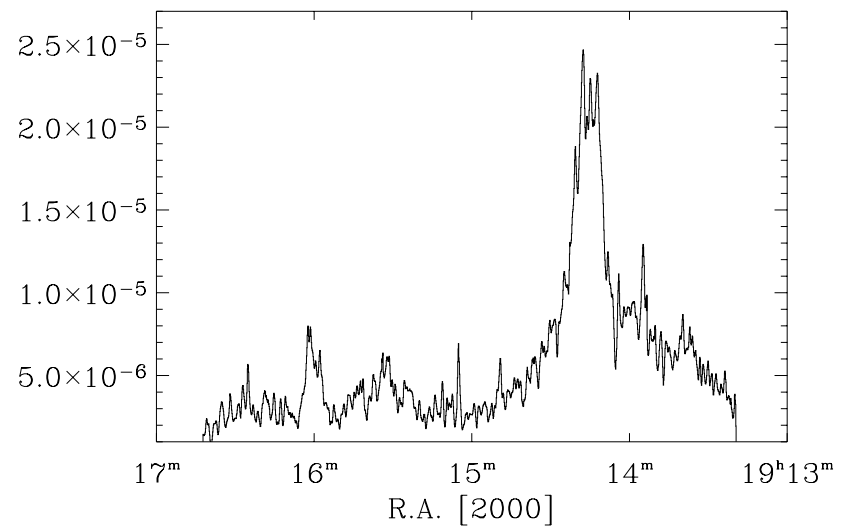

Fig. 2. Intensity cut along the dashed axis of Fig.1 joining the position of SS 433 and the center of the terminal shock. The maximum of the emission is about $35^{\prime}$ from the position of SS 433. The abscissa gives the counts/s in an image bin.

along this line. As in the earlier ROSAT observations (Paper I) the maximum of the emission appears at about $35^{\prime}$ from SS 433. The outer structures of the terminal shock are much weaker. Interestingly, the intensity of emission does not start abruptly at a certain position away from SS 433 (as expected from a standing shock) but increases already along the propagation direction of the jet before jumping to its maximum. Note that the figure does not provide an exact quantitative measure of the emission as raw data over the whole energy band from the PN and the MOS cameras, which have different energy dependent spectral responses, were merged.

As W50 is situated close to the galactic plane towards the galactic center $\left(l \sim 40^{\circ}, b=-3^{\circ}\right)$ we find a high background of point like sources in the fields of view. The brightest of them are either stars or optically unidentified and we will not further discuss them here.

\section{The eastern jet}

The most prominent features in Fig. 1 are the bright emission region at $\sim 19^{\mathrm{h}} 14^{\mathrm{m}} 12^{\mathrm{s}} .4$, which seems to be the termination of the jet coming into the picture from SS 433 (situated about 21 arcmin outside the picture on the right-hand side), and the much weaker fragmented structures of a "terminal shock" in the eastern "ear" of W50. The initially hot $(k T \gtrsim 10 \mathrm{keV})$, thermally radiating jets emitted from SS433 cool to temperatures below $10^{5} \mathrm{~K}$ on typical length scales of $\sim 10^{12} \mathrm{~cm}$ (see Brinkmann et al. 1991), emit the optical "moving" lines at distances $\sim 10^{15} \mathrm{~cm}$, show strong radio emission $(\gtrsim 0.5 \mathrm{Jy}$ at $5 \mathrm{GHz}$ with spectral index $\alpha=-0.6$ ) on scales from $10^{14}-10^{17} \mathrm{~cm}$ (cf. Vermeulen 1993, for a review), then remain "invisible" until they show up in X-rays again $\sim 15$ arcmin from SS433, i.e., at distances $\gtrsim 6.5 \times 10^{19} \mathrm{~cm}$. An insight into the physical mechanisms leading to this transfer of the kinetic energy of the jets into radiation of different frequencies, at different distances from the source, is certainly of great interest for our understanding of the jet phenomenon in general.

\subsection{The bright knot}

Using the procedures mentioned before for the production of overlaid pictures we created images in various energy ranges and formed an RGB - image, shown in Fig. 3. The "red" energy band was $0.5-1 \mathrm{keV}$, the "green" band $1.0-2.0 \mathrm{keV}$ and the "blue" band covered the energies $2.0-12 \mathrm{keV}$. (See Fig. 8 for the individual "soft" and "hard" images.)

The lenticular structure of the bright spot is clearly hard emission and this hard emission extends further to the right, 


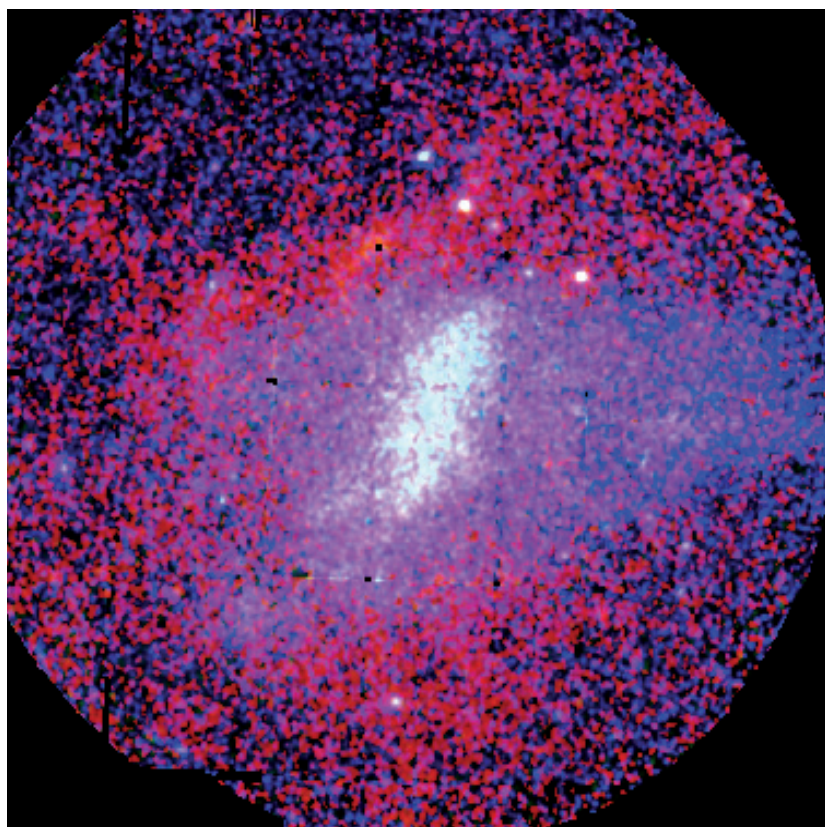

Fig. 3. RGB image of the bright knot from merged $\mathrm{PN}$ and MOS images.

towards SS 433. At lower energies the bright spot becomes diffuse, losing its well-defined shape and filling a large part of the central image (red colors). At the lowest energies the jet emission on the right side disappears. Also visible is the clearly reduced emission region in the north-eastern part of the image, the region outside the radio contours of W50. The emission filling the interior of the radio remnant is rather soft; at energies $\gtrsim 2 \mathrm{keV}$ the separation of the surface brightness between inside and outside W50 disappears. However, the low statistics of the signal at higher energies does not permit any quantitative analysis.

\subsubsection{Spectral analysis}

For the spectral fits we first extracted for all detectors the source photons from an ellipsoidal region coinciding with the brightest lenticular part in Fig. 3. Background spectra were extracted from the recast, renormalized blank-sky backgrounds as detailed above in Sect. 2.

Previous spectral analyses of this region favored a nonthermal power law spectrum (Yamauchi et al. 1994, Paper I). Figure 4 shows the result of a simultaneous fit of the PN and MOS detectors with an absorbed power law in the $0.6-8 \mathrm{keV}$ energy band. The fit resulted in a slope of $\Gamma=2.17 \pm 0.02$, an absorbing column density of $N_{\mathrm{H}}=(0.56 \pm 0.01) \times 10^{22} \mathrm{~cm}^{-2}$ with a reduced $\chi_{\text {red }}^{2}=1.085$ for 852 d.o.f. However, a fit with similar quality was achieved by a bremsstrahlung continuum model. The best fit parameters were $k T=3.98 \pm 0.11 \mathrm{keV}$, $N_{\mathrm{H}}=(0.41 \pm 0.01) \times 10^{22} \mathrm{~cm}^{-2}$ with a reduced $\chi_{\text {red }}^{2}=1.076$ (852 d.o.f.).

In both cases there were small residuals resembling weak emission lines in the soft energy band, for example $\gtrsim 0.9 \mathrm{keV}$ (Ne IX or Fe XIX) in Fig. 4. We therefore repeated the fit, first fitting a power law to the $2-8 \mathrm{keV}$ band data only. The extrapolation of the model showed some weak excess emission at low energies. We then fixed the power law parameters and fitted the total spectrum by adding a thermal (MEKAL) emission model. The fit resulted in a $\chi_{\text {red }}^{2}=1.038$ (for 853 d.o.f.) with best fit parameters of $\Gamma=2.41 \pm 0.09, k T=0.20 \pm 0.02 \mathrm{keV}$ and the galactic absorption increased to $N_{\mathrm{H}}=(1.07 \pm 0.24) \times 10^{22} \mathrm{~cm}^{-2}$.

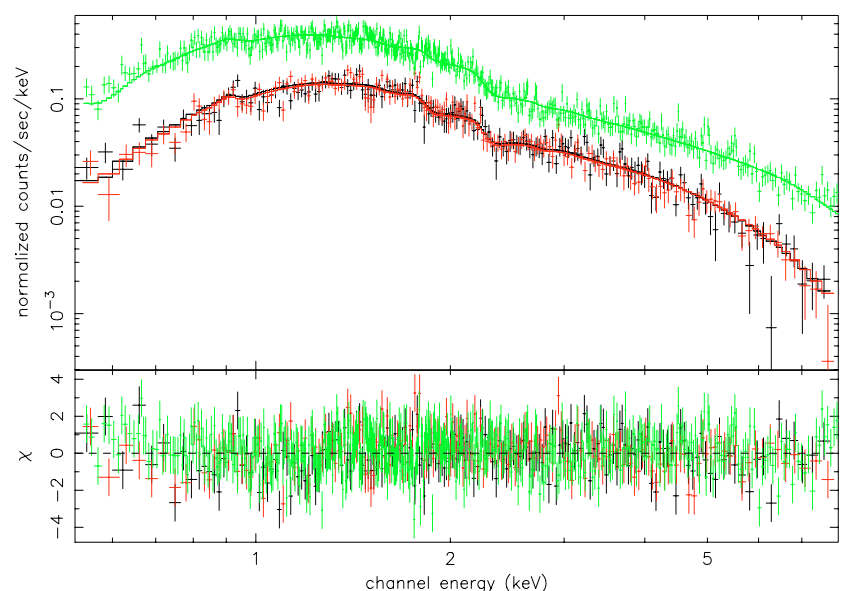

Fig. 4. Plot of the power law fit (upper panel) and residuals (lower panel) to the joint PN + MOS data in the 0.6-8 keV energy band for the center of the bright knot.

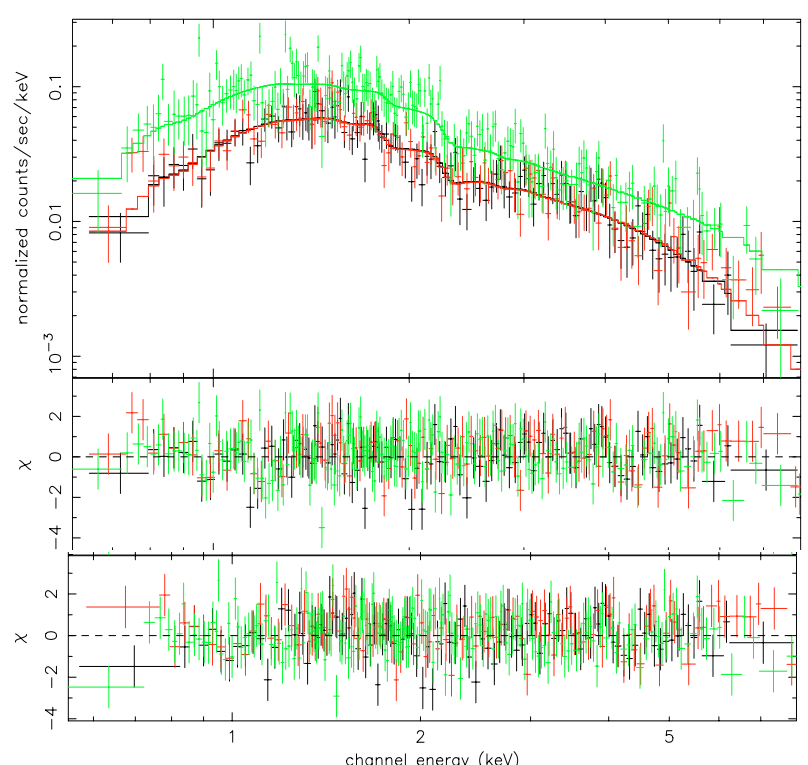

Fig. 5. Plot of a power law fit (upper panel) and residuals (middle panel) to the joint PN + MOS data in the $0.6-8.0 \mathrm{keV}$ energy band jet region. Bottom panel: residuals of a power law plus MEKAL model fit to the same data.

The contribution of the thermal component to the $0.2-2 \mathrm{keV}$ flux with $\sim 9.9 \times 10^{-12} \mathrm{erg} \mathrm{cm}^{-2} \mathrm{~s}^{-1}$ is of the same magnitude as the power law component of $\sim 6.6 \times 10^{-12} \mathrm{erg} \mathrm{cm}^{-2} \mathrm{~s}^{-1}$.

As there were still some indications for residuals in the emission line regions we fitted the non-equilibrium model VNEI in XSPEC instead of the MEKAL model in addition to the power law. The quality of the fit improved slightly $\left(\chi_{\text {red }}^{2}=1.022(850\right.$ d.o.f.)), the temperature remained similar, $k T=0.22 \pm 0.01$, and the ionization time $n_{\mathrm{e}} \times t=8.9 \times 10^{12} \mathrm{~s} \mathrm{~cm}^{-3}$ demonstrates that the plasma is nearly in ionization equilibrium. Leaving the elemental abundances as free parameters resulted in values compatible with the cosmic values inside the errors, which are quite large due to the limited data quality.

To investigate the jet emission outside the pronounced lens structure we extracted photons from a rectangular region on the jet at the right hand boundary of the image (Fig. 3) which seems to show only hard jet emission from the RGB colors. Figure 5 shows in the upper two panels the power law fit and the residuals to the joint PN and MOS data and the residuals. The power 


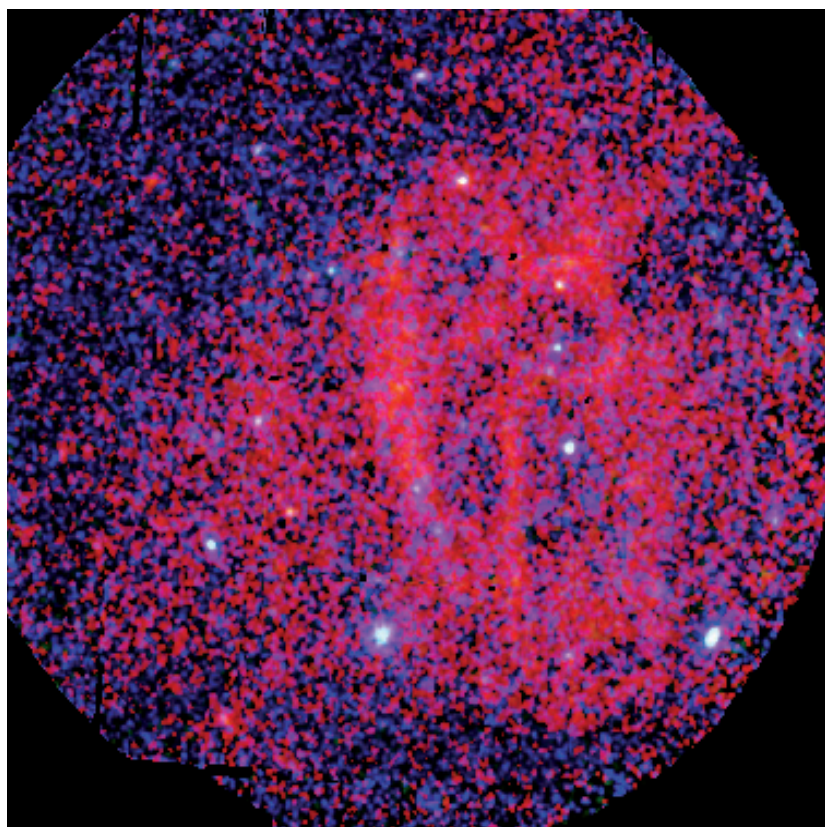

Fig. 6. RGB image of the terminal shock region from merged PN and MOS images.

law model provides an excellent fit to the data $\left(\chi_{\text {red }}^{2}=0.963\right.$ for 508 d.o.f) with $\Gamma=1.85 \pm 0.06$ and an absorbing column density of $N_{\mathrm{H}}=(0.62 \pm 0.04) \times 10^{22} \mathrm{~cm}^{-2}$. To account for some smaller residuals in the soft band we first fitted again a power law at higher energies then added an extra MEKAL model, obtaining a fit with nearly identical quality $\left(\chi_{\text {red }}^{2}=0.977\right.$ (514 d.o.f. $\left.)\right)$ over the whole energy band. The temperature was similar to that of the fit to the "lens" region $(k T=0.22 \pm 0.01) \mathrm{keV}$, the hard power law slope is steeper $(\Gamma=2.15 \pm 0.56)$ compared to the simple power law fit, and the amount of absorption increased $\left(N_{\mathrm{H}}=(1.38 \pm 0.04) \times 10^{22} \mathrm{~cm}^{-2}\right)$. Thus, the quality of the data does not rule out a thermal component in addition to the power law, but does not require it either.

\subsection{The terminal shock}

Figure 6 shows the RGB-image of the region of the terminal shock. Two features are worth noticing: first of all the diffuse emission is really restricted to the area inside the corresponding radio contours of the W50 remnant (see Fig. 1), outside of which there is no emission to the level of the background fluctuations. Secondly, the bright rim, also visible in the radio maps, appears to be just the front part of a ring-like emission structure which fills the whole circumference of the conically converging remnant. Assuming that this structure is a perfect ring (doughnut) its inclination to the line of sight must be of the order of $30^{\circ}$, not quite consistent with the inclination of the SS 433 system of $\sim 78^{\circ}$.

The spectrum of the bright central rim is very noisy, possibly caused by the fact that due to its low surface brightness a relatively large extraction area has to be used which can include unresolved background sources. A simple continuum model (bremsstrahlung, power law) does not fit the data adequately. A power law plus a MEKAL model accounts for some of the "line like" residuals, but the fit is unacceptable $\left(\chi_{\text {red }}^{2}=2.0\right.$ for 226 d.o.f.) for the PN.

In Fig. 7 we show the fit to the joint PN and MOS data of the bright filament with a power law plus VNEI model in the

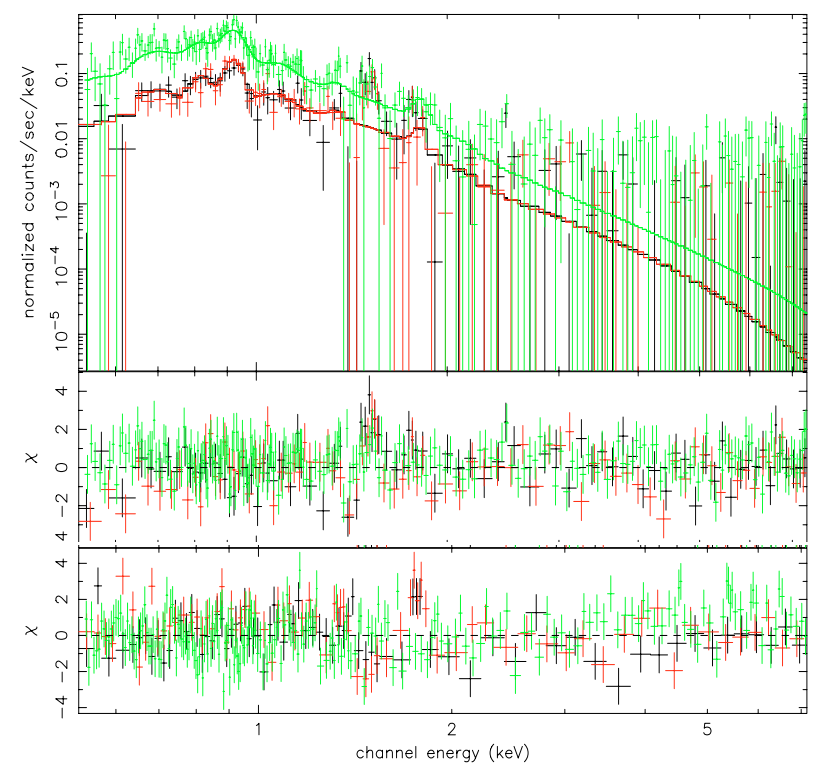

Fig. 7. Plot of the power law plus VNEI model fit (upper panel) and residuals (lower panels) to the joint PN + MOS data in the $0.5-7.5 \mathrm{keV}$ energy band for the region of the bright filament in Fig. 6. Upper two panels: fit and residuals with blank sky background. Lower panel: residuals from the fit with the local background.

0.5-7.5 keV energy band which resulted in a $\chi_{\text {red }}^{2}=1.07$ for 648 d.o.f. The hard power law has a slope of $\Gamma=4.5 \pm 1.1$, the temperature of the VNEI model is $k T=0.28 \pm 0.01 \mathrm{keV}$, the ionization time $n_{\mathrm{e}} \times t=(2.9 \pm 1.1) \times 10^{10} \mathrm{~s} \mathrm{~cm}^{-3}$ and the fitted absorption $N_{\mathrm{H}}=(0.82 \pm 0.05) \times 10^{22} \mathrm{~cm}^{-2}$. Leaving the abundances free resulted in over-abundances of $\mathrm{Ne}, \mathrm{Mg}, \mathrm{Si}, \mathrm{Fe}$, and $\mathrm{Ni}$, although with values which are mostly consistent with solar abundances within the errors. The residuals clearly show the differences between the various instruments, especially at low energies.

It is obviously hard to separate the spectrum of the "ring" from a diffuse background which provides more signal than the source itself. Another source of uncertainty is the fact that the background is taken from a blank field accumulated from pointings at higher galactic latitudes, whereas the source is seen against a background about 3.0 degrees below the galactic plane. We therefore repeated the fit with a local background selected from just outside radio remnant from the current observation, i.e. the upper left region in Fig. 6. This fit is relatively bad, $\chi_{\text {red }}^{2}=1.38$ (479 d.o.f.) but the best fit parameters are roughly consistent with the above values inside their mutual errors: the power law slope is $\Gamma=2.29 \pm 0.50$, the temperature of the VNEI model is $k T=0.40 \pm 0.02 \mathrm{keV}$, the ionization time $n_{\mathrm{e}} \times t=(2.7 \pm 0.3) \times 10^{10} \mathrm{~s} \mathrm{~cm}^{-3}$ and the fitted absorption $N_{\mathrm{H}}=(0.38 \pm 1.63) \times 10^{22} \mathrm{~cm}^{-2}$. The fitted overabundances are similar as above, however with formally smaller errors which must be regarded with caution considering the unacceptable quality of the fit. The residuals of that fit are shown as the bottom panel of Fig. 7 .

A comparison of the two fits indicates that the data quality is insufficient to accurately constrain all of the model parameters: at higher energies (power law slope) as well as in the soft band (absorption) the fitted parameters depend strongly on the background subtraction.

We finally tried to determine the spectrum in the very eastern parts of W50, to the left of the bright ring. The signal is rather low and the fit residuals remain noisy even after all of the obvious faint point sources have been removed from the data. 


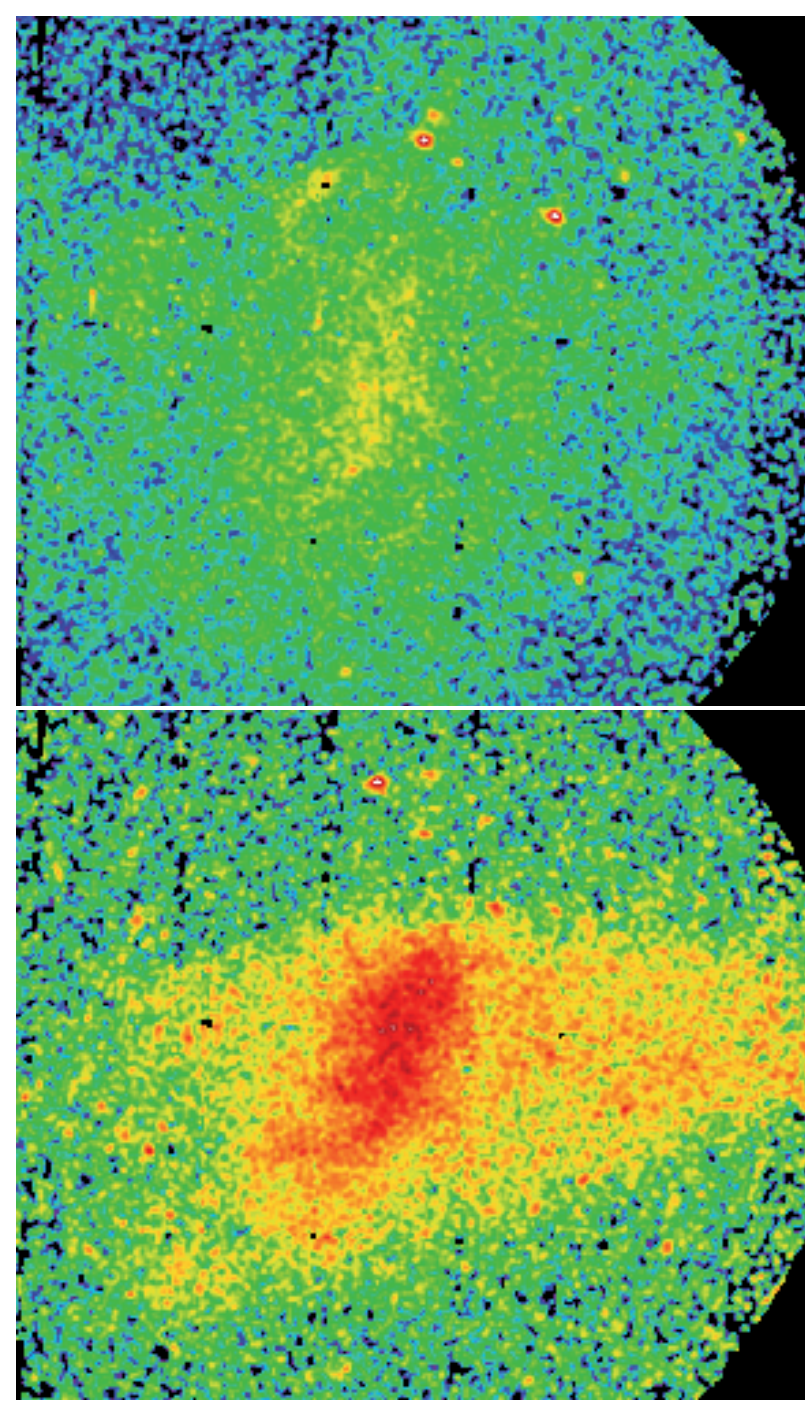

Fig. 8. Exposure and vignetting corrected summed PN+MOS images of the lenticular emission region in the $0.2-1 \mathrm{keV}$ (top) and $2-12 \mathrm{keV}$ (bottom) energy band. The image sizes are $8^{\prime} \times 7^{\prime}$ each.

No simple model fits the data but a power law with MEKAL model plus an extra line at $0.924 \pm 0.07 \mathrm{keV}$ results in an acceptable fit with $\chi_{\text {red }}^{2}=1.14$ for 520 d.o.f. The power law with $\Gamma=1.32 \pm 0.13$ dominates completely the emission of the thermal component $(k T=0.31 \pm 0.03 \mathrm{keV})-$ apart from some prominent emission lines.

\section{W50 and SS 433}

The radio remnant $\mathrm{W} 50$ has a very distinct shape consisting of a nearly spherical shell centered on SS433 plus two elongations, the "ears", in the direction of the jets. A commonly accepted model for the elongated morphology is the interaction between the SNR shell and the out-flowing jets. It should be mentioned that according to our understanding of the geometrical conditions of SS 433 the jet causing the eastern "ear" is pointing towards us. Thus the X-ray spectrum emitted from the jet is a tracer of the physical processes leading to the slowing down of the jet by transforming its kinetic energy into radiation.

\subsection{The morphology}

The X-ray brightness distribution reveals some details which are not in agreement with the simple shell - jet interaction described above. While the locus from SS 433 to the emission centroid of the terminal shock shows good symmetry with respect to the outer X-ray and radio emission, the lenticular structure and its axis of emission towards SS 433 certainly breaks this symmetry. Further, the angular extent of the X-ray jet (about $18^{\circ}$ as seen from SS 433) is much smaller than the precession angle of $\sim 40^{\circ}$ expected from the kinematic model. This indicates either some collimation of the precessing jets (Peter \& Eichler 1993) or is a consequence of the interaction between the jet and the SNR which generates secondary shock waves along the symmetry axis (Velázquez \& Raga 2000).

The lenticular emission structure has an angle of 20-30 degrees to the jet propagation direction, thus it cannot be a simple standing shock perpendicular to the jet axis.

There is steadily increasing emission, starting outside the right boundary of the XMM field of view, about 15 arcmin from SS 433 (see Fig. 6 of Paper I), building up to a very sharp and relatively narrow peak about 35-40 arcmin from SS 433 (see Fig. 2). This indicates that from distances $\gtrsim 6 \times 10^{19} \mathrm{~cm}$ from the compact source, the kinetic energy of the out-flowing matter is transferred to radiating relativistic electrons at an increasing rate until an "unstable" situation is reached at the emission peak, where suddenly a much larger fraction of the energy is dissipated. Further away from SS 433 the jet bends southwards towards the terminal shock, the emission dropping to a very low level before entering the region of the terminal shock itself.

At higher X-ray energies the boundary of the emission zone perpendicular to the jet direction is rather sharp, the intensity increases typically by a factor of $\sim 2.5$ and shows a flat top profile. At low energies the profile smears out and widens, as if the radiating electrons diffuse out of the acceleration zone. This can be seen in Fig. 8. In the hard 2-12 keV energy band (bottom figure) the emission region is sharply bounded as explained above and clearly pointing towards SS 433 . The soft $0.2-1 \mathrm{keV}$ image (top) shows only an extended diffuse emission region, centered on the lenticular structure, without any connection to SS 433 on the right, outside the images.

The transfer of kinetic energy of the relativistically flowing matter into radiation is obviously a complex process and it is not quite clear whether it is controlled "intrinsically" (i.e. just by the flow itself), or whether environmental conditions play a role as well. Note that the outer shape of the surrounding radio remnant W50 shows a very pronounced northern bay at a similar right ascension as the X-ray emission maximum $\left(\sim 19^{\mathrm{h}} 14^{\mathrm{m}}\right)$, which does not have a counterpart on the southern side.

At the outermost eastern boundary of W50 the X-ray and the radio structures show an astonishingly similar appearance, indicating the interaction of a "terminal shock" of the jet and the circumstellar medium. The interior of the radio remnant is filled with X-ray emitting material, and the spatial coincidence of X-ray and radio emission suggests that the physical conditions of the terminal shock region are very similar to those found at the outer shocks of "ordinary" supernova remnants. Interestingly, the prominent X-ray emission seems to form a ring-like structure confined by the outer boundary of the radio remnant. This indicates that the final jet flow might still have some kind of hollow-cone morphology. 


\subsection{The $X$-ray spectrum}

XMM-Newton has for the first time the sensitivity and the wide band pass to study in more detail the spectral behavior of the precessing jet interacting with its ambient medium. However, the photon statistics still do not allow accurate fits of individual small spatial structures visible in the data. Fortunately, there are no indications for drastic spectral changes occuring on small spatial scales (e.g., Figs. 3 and 4).

A simple power law model provides an excellent representation of the spectrum of the main bright lenticular area of Fig. 3 . The obtained slope of $\Gamma=2.17 \pm 0.02$ is in the range commonly found for non-thermal supernova remnants like in the Crab. A similar power law is seen in the outer, terminal shock region although there the low statistical significance of the high energy data does not allow a very precise determination of the slope.

It must further be noticed that the bright spot can be equally well fitted with a bremsstrahlung model with $k T=3.98 \pm$ $0.11 \mathrm{keV}$, a temperature in agreement with previous findings from ASCA (Yamauchi et al. 1994), ROSAT (PaperI) and Einstein (Watson et al. 1983). However, we tend to believe that this model is merely a "statistically acceptable" numerical representation of the spectral shape and not a correct description of the physical emission conditions, in particular, as the data show no indications of $6.7 \mathrm{keV}$ iron line emission, which would be expected at these temperatures.

In both cases, power law or simple bremsstrahlung, we find in the soft band spectral residuals resembling well known emission lines. While in the lenticular spot these features are relatively weak they are clearly dominant in the terminal shock region. Adding to the power law a MEKAL model resulted in temperatures of $\sim 0.2-0.3 \mathrm{keV}$ for the thermal models, but the residuals still showed some line features which were not accounted for in this thermal ionization equilibrium model. Using the nonequilibrium model VNEI instead of MEKAL improved the quality of the fits without significant change to the basic physical parameters. For the lenticular region the temperature remained at $k T=0.22 \pm 0.01 \mathrm{keV}$, for the terminal shock we obtained a $k T=0.28 \pm 0.01 \mathrm{keV}$; in both cases the ionization time scales were slightly below equilibrium ionization conditions and there are indications for higher than solar abundances of $\mathrm{S}, \mathrm{Si}, \mathrm{Fe}$ and $\mathrm{Ni}$.

In the fits with simple continuum models the values for the galactic absorption turned out to be $N_{\mathrm{H}} \sim 0.5 \times 10^{22} \mathrm{~cm}^{-2}$, i.e., smaller than the values found for SS 433 itself (PaperI, Brinkmann et al. 2005). This extra absorption towards the central source SS 433 might quite well be related to the "equatorial ruff" detected in the radio band (Blundell et al. 2001). However, if we add a low energy thermal model to the power law, the fitted absorption can reach values $N_{\mathrm{H}} \sim 10^{22} \mathrm{~cm}^{-2}$, similar to those of SS 433 itself. The exact value depends on the complex interplay between the normalization of a soft thermal model and the absorption in a relatively narrow soft energy band. It is further affected by the choice of the background and partly on the instrument under discussion.

For both pointings we tried spectral fits to the regions away from the prominent features; i.e., where the jet enters the field of view from the right of Fig. 8, and at east (left in Fig. 6) of the ring-like terminal shock. For the latter the signal is very low but both a power law and thermal emission are required by the data. The contribution of the power law component is rather high which might be due to the fact that the extended source covers the projection of the whole final outer shock region of W50. The spectrum of the jet, entering the field of view from the right in
Fig. 8 is predominantly of power law origin, similar to that of the bright lenticular region. The addition of a thermal model does not improve the fit significantly, but cannot be ruled out either.

\section{Physical estimates}

Compared to previous observations we have been able to better constrain the spectral parameters of the eastern jet with these XMM-Newton observations.

Using the bright lenticular structure for the parameter estimates, we find from the fits to the data a number density of the thermally radiating particles of $0.2 \lesssim n_{\mathrm{e}} \lesssim 0.7 \mathrm{~cm}^{-3}$. This, and the following estimates are based on the assumption of constant density in the emission regions. Clumping of the gas will lead to a over-estimation of the electron densities and lower the required total mass and energy of the gas. Additionally, the total volume of the emission region might be larger by a factor of a few than the chosen extraction regions for the fits.

Assuming cosmic abundances this thermally radiating gas has a cooling time scale of $\sim 10^{7}$ yr. The ellipsoidal (in 2dimensional projection lenticular) emission region has a volume of $\sim 6.5 \times 10^{57} \mathrm{~cm}^{3}$, containing $2 \times 10^{57}$ particles with a total thermal energy content of $5 \times 10^{48} \mathrm{erg}$.

The kinetic outflow energy of the jets of SS 433 are of the order of $10^{39}-10^{40} \mathrm{erg} \mathrm{s}^{-1}$ following early estimates and the numerical modeling of the jet emission (for example Brinkmann et al. 1991, Brinkmann \& Kawai 2000). With its known outflow velocity of $\sim 0.26 \mathrm{c}$ a jet thus supplies about $\lesssim 2 \times 10^{44}$ particles per second into the remnant. While the energy input from the jets over a life time of the system of $\sim 5 \times 10^{4} \mathrm{yr}$ is obviously far exceeding the thermal energy content of the gas the number of thermal particles in the remnant must be supplied by other means. If the mass loss rate of the primary of the SS 433 binary system, estimated to be $2-3 \times 10^{-4} M_{\odot} \mathrm{yr}^{-1}$ (Begelman et al. 1980; Fuchs et al. 2006) operates over the life time of the system a total number of $\sim 2 \times 10^{59}$ particles would have been injected into the remnant, fully sufficient to account for the density of thermal particles in W50. Thus it seems that the gas mass in the remnant is supplied by the wind from the binary system whereas the energy comes predominantly from the out-flowing jets.

The X-ray luminosities from the outer jets represent in any case only a small fraction of the available energy supply by SS 433: the $0.5-10 \mathrm{keV}$ luminosity in the power law component of the bright emission is $L_{\mathrm{pl}} \gtrsim 2 \times 10^{34} \mathrm{erg} \mathrm{s}^{-1}$; the total luminosity of the soft thermal emission might be up to a factor of 2-3 higher. Even if the total emission region is a few times larger than the region extracted for the fits, the total luminosity amounts only to $10^{-5}-10^{-4}$ of the kinetic energy of the jets.

Assuming equipartition between the energy in the magnetic field and the relativistic electrons, the deduced luminosity of the power law emission yields an estimate for the magnetic field strength of $B \sim 2-4 \times 10^{-6} \mathrm{G}$ (Watson et al. 1983; Yamauchi et al. 1994). The number density of the relativistic electrons is $\sim 4 \times 10^{-14} \mathrm{~cm}^{-3}$, the total energy content in the magnetic field and the electrons is $\sim 10^{45}-10^{47} \mathrm{erg}$. Thus, the relativistic electrons as well represent only a small fraction of the kinetic energy deposited by the jets into the remnant over its estimated life time.

The most challenging physical question is how the directed kinetic energy of the jet is transferred to the relativistic electrons and then into radiation. The simple picture of the jet being decelerated by the collision with the supernova shell (Murata \& Shibazaki 1996; Velázquez \& Raga 2000) can hold only for the terminal shock in the outermost eastern "ear". 
Most of the emission, however, occurs further in, about 35 arcmin away from SS433 and the X-ray intensity profile along the jet axis indicates a gradual growth of the energy transfer rate, starting at $\sim 6 \times 10^{19} \mathrm{~cm}$ from SS 433. If there the precessing jet would still follow an undisturbed ballistic motion it would have formed a conical spring with a separation of $\gtrsim 10^{17} \mathrm{~cm}$ between the turns which are only $\sim 1.5 \times 10^{15} \mathrm{~cm}$ thick. The radius of the "spring" at this distance from SS 433 would be about $2.5 \times 10^{19} \mathrm{~cm}$, which is $\sim 5.5$ at the distance of $5 \mathrm{kpc}$ for SS 433 . This is certainly not observed which means that up to this distance a re-collimation of the jet must have already taken place. Further, early 3-dimensional hydrodynamical simulations of the precessing jet (Brinkmann \& Müller 1998) showed that already at distances $\lesssim 10^{17} \mathrm{~cm}$ the jet develops instabilities which lead to a dispersion of the matter of the ordered flow. If thus the material of the conical "spring" blends together, forming a hollow cone, and if most of the interior of this cone is evacuated, the jet might be re-collimated hydrodynamically (Peter \& Eichler 1993). If the above discussed thermal gas fills the remnant its pressure would be sufficient to lead to a jet re-collimation at a distance of $\sim 4.9 \times 10^{19} \mathrm{~cm}$ (Eichler 1983) in excellent agreement with the distance of the onset of X-ray emission from SS 433.

The flow then obviously diverges again with an opening angle of $\sim 18^{\circ}$ and a steadily increasing X-ray flux. The process of transferring the kinetic energy of the jet matter to relativistic particles is unknown, it might be the result of a Kelvin-Helmholtz instability or some other instability related to the motion of the jet material through the surrounding medium (Rose et al. 1984; Beall 1990). In any case the rate of energy transfer seems to grow until it reaches a "critical" level, about $1.5 \times 10^{20} \mathrm{~cm}$ from SS 433 where the X-ray emission suddenly increases by a factor of $\sim 2.5$, lasts for about $10^{19} \mathrm{~cm}$ and then fades away rapidly.

The fact that this emission structure is not perpendicular to the jet propagation direction (as well as perhaps the sudden onset of the enhanced X-ray emission itself) might be related to a substantial gradient in the initial distribution of the interstellar matter to the north-east of W50: the radio shell exhibits a big "dent" in its outer rim, the northern part of the jet flow is braked, leading to a re-direction of the main flow and an oblique lenticular emission zone. That from here on the flow characteristic changed markedly can be seen from the drastically reduced $\mathrm{X}$-ray emission towards the terminal shock.

\section{Discussion and conclusions}

We have presented the data of two XMM EPIC observations of the eastern jet of SS 433, one pointed on the bright emission region about $35^{\prime}$ from SS 433, the second on the terminal shock region in the eastern "ear" of W50. The spectra can be best fitted with the superposition of two components: a power law with photon index $\Gamma=2.17 \pm 0.02$ and a non-equilibrium ionization thermal component with temperatures around $k T \sim 0.3 \mathrm{keV}$. In the bright region the power law clearly dominates, in the terminal shock region both components are of similar strength. The exact strength of the thermal component is hard to determine from the current data due to the limited data quality and "technical" difficulties with the spectral analysis at lowest energies.

The soft X-ray emission seems to fill most of the volume of the eastern radio remnant and no X-ray emission is seen outside the lowest radio contours to the limit of the statistical background fluctuations.

In the inner parts of the remnant, between SS 433 and the bright lenticular emission region, the jet supplies energy to its surroundings via some unknown mechanism, obviously generating relativistic electrons which then diffuse into larger regions of the volume of W50. The observed shape of the emission volume argues for an initial re-focusing of the precessing jet in the innermost $\sim 6 \times 10^{19} \mathrm{~cm}$. The energy transfer to relativistic electrons then increases, leading to enhanced emission along the jet propagation axis with an opening angle of $\sim 20^{\circ}$. At a distance of $\sim 35^{\prime}$ from SS 433 the emission brightens suddenly, forming at higher X-ray energies a lenticular shape, oblique to the jet axis. It is proposed that this is caused by the interaction with some exterior matter distribution which is also responsible for the deformation of the outer shape of the radio remnant in this region.

Far out in the eastern "ear" the X-ray emission forms a ring like terminal shock indicative for the braking of a hollow cone flow. The spatial coincidence of X-ray and radio emission suggests that the physical conditions of the terminal shock region are very similar to those found at the outer shocks of "ordinary" supernova remnants. The low temperature fitted for the thermal component implies that this terminal shock cannot be very strong, i.e., the jet flow must have been considerably slowed down from its initial $0.26 \mathrm{c}$. Where and how this actually happens, remains unclear.

The total energy radiated from the jet is only a small fraction $\left(\lesssim 10^{-4}\right)$ of the kinetic energy flux in the jets, integrating the synchrotron spectrum from the radio- to the hard X-ray band. Further, the estimated total energy of the inferred relativistic electrons and the thermal gas is much smaller (of the order of $\sim 10^{-4}$ ) than the energy injected into the remnant by the jets over its estimated life time of $\gtrsim 10^{4}$ years. The faint extended $\mathrm{X}$-ray emission from large regions inside the remnant requires additional gas which can be easily supplied by the strong wind of the SS 433 binary system. The injected energy is transported away from the sites of the interaction of the jet with its surroundings and the sharp boundaries of the emission regions of the hard $\mathrm{X}$-rays can provide important information about the cooling/diffusion time scales in the system. A substantial fraction of the jet's kinetic energy input might further be consumed to accelerate the matter in the "ears". Numerical modeling is obviously required to estimate the efficiency of transferring the jet's kinetic energy into relativistic particles, and to understand where the remaining energy is going.

Finally, another interesting result is related to SS433 itself. For most spectral fits, SS 433 itself shows higher absorption than the X-ray lobes. The excess absorption of the central object is of the order of $\Delta N_{\mathrm{H}} \sim(4-5) \times 10^{21} \mathrm{~cm}^{-2}$. This might be caused by extra intervening material, e.g., from a "cocoon" of stellar wind material blown from the primary. Such an "equatorial ruff" has also been detected in the radio band, and has been interpreted in terms of a non-isotropically emitted outflow of matter from the binary.

Acknowledgements. This work is based on observations with XMM-Newton, an ESA science mission with instruments and contributions directly funded by ESA Member States and the USA (NASA). We thank Wolfgang Pietsch and Michael Bauer for their help with the technical details of merging the images of the two observations.

\section{References}

Arnaud, M., Neumann, D., Aghanim, N., et al. 2001, A\&A, 365, L80

Beall, J. H. 1990, in Physical Processes in Hot Cosmic Plasmas, ed. W. Brinkmann, A. C. Fabian, \& F. Giovannelli (Dordrecht: Kluwer), 341

Begelman, M. C., Sarazin, C. L., Hatchett, S. P., McKeee, C. F., \& Arons, J. ApJ, 238,722

Blundell, K. M., \& Bowler, M. G. 2004, ApJ, 616, L159 
Blundell, K. M., Mioduszewski, A. J., Muxlow, T. W. B., Podsiadlowski, P., \& Rupen, M. P. 2001, ApJ, 562, L79

Brinkmann, W., \& Müller, E. 1998, in Astrophysical jets, ed. S. Massaglia, G. Bodo (Amsterdam: Gordon \& Breach), 211

Brinkmann, W., Kawai, N., \& Matsuoka, M. 1989, A\&A, 218, L13

Brinkmanni, W., Kawai, N., Matsuoka, M., \& Fink, H. H. 1991, A\&A, 241, 112

Brinkmann, W., Aschenbach, B., \& Kawai, N. 1996, A\&A, 312, 306

Brinkmann, W., Kotani, T., \& Kawai, N. 2005, A\&A, 431, 575

Dubner, G. M., Holdaway, M., Goss, W. M., \& Mirabel, I. F. 1998, AJ, 116, 1842

Ehle, M., Breitfellner, M., Gonzales Riestra, R., et al. 2005, XMMNewton Users Handbook, http://xmm.vilspa.esa.es/xmm _user_support/documentation/uhb/

Eichler, D. 1983, ApJ, 272, 48

Fuchs, Y., Koch Miramond, L., \& Abraham, P. 2006, A\&A, 445, 1041

Hjellming, R. M., \& Johnston, K. J. 1981, ApJ, 246, L141

Kawai, N., Matsuoka, M., Pan, H. C., \& Stewart, G. C. 1989, Publ. astr. Soc. Pac. 41, 491

Kawai, N., Yuan, W., \& Kotani, T. 1994, in New Horizon of X-ray Astronomy, ed. F. Makino, T., Ohashi (Tokyo: Universal Academy Press), 67

Kotani, T., Kawai, N., Matsuoka, M. \& Brinkmann, W. 1996, PASJ, 48, 619

Kemp, J. C., Henson, G. D., Kraus, D. J., et al. 1986, ApJ, 305, 805
Margon, B. 1984, Ann. Rev. Astr. Astr., 22, 507

Marshall, H. L., Canizares, C. R., Heinz, S., et al. 2005, in Proc. of Relativistic Astrophysics and Cosmology, Einstein's Legacy, ed. B. Aschenbach, V. Burwitz, G. Hasinger, \& B. Leibundgut (Berlin: Springer Verlag), 285, in press

Migliari, S., Fender, R., \& Méndez, M. 2002, Science, 297, 1673

Migliari, S., Fender, R., Blundell, K. M., Méndez, M., \& van der Klis, M. 2005, MNRAS, 358, 860

Peter, W., \& Eichler, D. 1993, ApJ, 417, 170

Pietsch, W., Misanovic, Z., Haberl, F., et al. 2004, A\&A, 426, 11

Read, A. M., \& Ponman, T. J. 2003, A\&A, 409, 395

Rose, W. K., Guillory, J., Beall, J. H., \& Kainer, S. 1984, ApJ, 280, 550

Velásquez, P. F., \& Raga, A. C. 2000, A\&A, 362, 780

Vermeulen, R. 1993, in Astrophysical Jets, ed. R. Burgarella, M. Livio, \& C. O'Dea (Cambridge: Cambridge Univ. Press), 241

Watsoni, M. G., Willingale, R., Grindlay, J. E., \& Seward, F. D. 1983, ApJ, 273, 688

Watson, M. G., Stewart, G. C., Brinkmann, W., \& King, A. R. 1986, MNRAS, 222, 261

Yamauchi, S., Kawai, N., \& Aoki, T. 1994, Pub. Astr. Soc. Jap., 46, L109

Yuan, W., Kawai, N., Brinkmann, W., \& Matsuoka, M. 1995, A\&A, 297, 451 